\title{
Phishing Detection using Machine Learning Techniques
}

\author{
Santhi H, Supraja, Basi Reddy A,Sailaja G
}

\begin{abstract}
A phishing email is legal-looking email which may be planned with trap the beneficiary under trusting that same as certifiable email, Furthermore Possibly uncovers delicate data or downloads pernicious injecting codes through clicking ahead pernicious joins held in the particular figure of the email. There would various provisions receptive to phishing ID number. However, Dissimilar to predicting spam there need aid exactly couple of focuses that ponder machine Taking in routines to anticipating phishing. In this paper an information set is used to arrange those phishing identification those display dataset employments choice tree to predicting phishing messages. We would be setting off should investigate consideration of extra variables of the data set, which might enhance the predictive correctness of classifiers. For example, analysing email headers need demonstrated will move forward the prediction ability What's more diminishing those misclassification rate about classifiers.
\end{abstract}

\section{INTRODUCTION}

There is no particular time to end representation to phishing. However, a enormous portion descriptions choose that those objective of a phishing trick may be should takeindividuals' particular secret data. The networking of the strike might shift reliant on the trap setup. For example, Pharming may be a kind from claiming phishing, the place the attacker misdirects clients with fakelocations alternately proxy servers, commonly through space name framework (DNS) capturing or poisoning [3]. In this the event Attacker might take victimized people's data Toward securing a space should be phishing website without sending twisted messages. By email remains the A large portion positive position vehicle to phishing. Those plenitudes for off-therack mass mailing devices (dubbed as mailers) simplifies those vocation of phishers Also Assistance Previously, sending an immense number for messages should anextensive amount about victims. Investigations demonstrate a persistent expansion over phishing exercises and additionally those related pamper. On 2003 immediate phishing-related passing banks and MasterCard issuers might have been evaluated Finally Bob's examining $\$ 1.2$ billion which grew with $\$ 2$ billion on over 2,800 doctor look tasks led from April 1, 2009 to March 31, 2010. Over January 2007, the absolute number for interesting phishing reports submitted of the Anti-Phishing working one assembly (APWG) might have been 29; 930. This will be

Revised Manuscript Received on September 14, 2019.

Santhi H, School of Computer Science and Engineering Vellore Institute of Technology, Vellore, Tamilnadu, India

Supraja, School of Computer Science and Engineering Vellore Institute of Technology, Vellore, Tamilnadu, India

Basi Reddy A, Department of CSE, S.V. College of Engineering, Tirupati, A.P, India

Sailaja G, Department of CSE, S.V. Engineering College, Tirupati, A.P, India name to a website Also redirecting that website's movement

the most elevated amount from claiming reports recorded Toward the APWG [3]. Contrasted with the past top for June 2006, those number for submitted reports expanded by $5 \%$. Despite the fact that there are a few results recommended Furthermore actualized to identification What's more anticipation from claiming phishing strike. The greater part for tolerate starting with unsuitable levels from claiming false positives or missed identification.

A few machine Taking in techniques including logistic relapse (LR), order Also relapse Trees (CART), Bayesian added substance relapse Trees (BART), help vector Machines (SVM), irregular Forests (RF), What's more Neural Networks (NNet) need aid used to identify phishing. As stated by those APWG there are, previously, general, three primary classifications for phishing

Furthermore duplicity protective mechanisms; detective, preventive, and restorative results [3]. These Classes Alsotheir relating subcategories would summarized Previously, table 1. In the accompanying we furnish a short depiction from claiming few accessible phishing identification systems. In we depict against phishing toolbars. Afterwards, we survey two Scrutinize investigations that apply machine Taking in On phishing identification.

\subsection{Anti-Phishing Toolbars}

Anti-phishing toolbars are ubiquitously accessible Furthermore regularly utilized by credulous alternately nontechnical PC clients will assistance allay those phishing

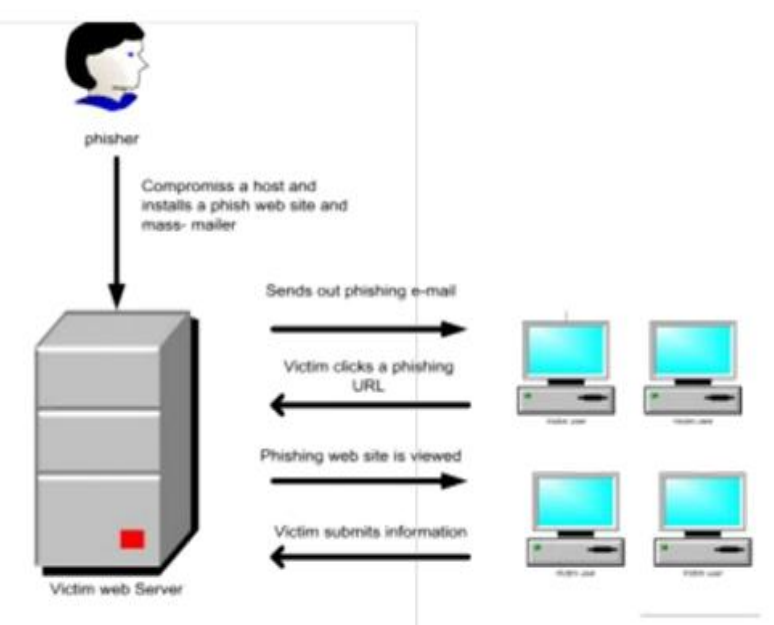

Fig:1 phishing E-mail process

Published By: 
issue. In spite of these toolbars help relieve those problem, huge numbers researchinvestigations need exhibited that incapability from claiming such systems.

Table:1 Phishing and Fake solutions

\begin{tabular}{|l|l|l|}
\hline $\begin{array}{l}\text { Detective } \\
\text { solutions }\end{array}$ & $\begin{array}{l}\text { Preventive } \\
\text { solutions }\end{array}$ & $\begin{array}{l}\text { Corrective } \\
\text { solutions }\end{array}$ \\
\hline 1.Monitor & 1.Authenticatio & 1. Site \\
s account & n & takedown \\
life cycle & 2. E-mail & Forensics \\
2. Brand & Authentication & and \\
Monitorin & Patch and & investigatio \\
g & change & n \\
3.Disables & management & \\
web & 3. Web & \\
duplicatio & application & \\
n & security & \\
4.Perform & & \\
s content & & \\
filtering & & \\
Anti- & & \\
malware & & \\
Anti-spam & & \\
\hline
\end{tabular}

\section{METHODOLOGY}

Anti-phishing approaches Should beat the restrictions from claiming conventional, PC security masters created toolbar visualization techniques, for example, such that Ebay_Guard [10], Netscape [23], Netcraft [22], McAfee site counselor [16], parody adhere [28] "around others. A security toolbar is as a rule installed inside a web program Furthermore its part may be on uncover sure security majority of the data of the end- client over possibility internet risks, for example, such that phishing strike. To instance, parody adhere toolbar shows a famous of the enduser. At this famous may be green, this is an implication that it may be protected to scan and, point when the symbol will be red, this demonstrates that those websites doesn't have a place with Ebay alternately Paypal. Nevertheless, the totallyspread from claiming visualization techniques available, their phishing identification rate may be low [17]. instance,contrasted three toolbars utilizing security indicators Furthermore indicated that know for them were unabated will keepphishing exercises. Those creators additionally indicated that pop-up messages appear that's only the tip of the iceberg positive position methodology to battle phishing over toolbars, since pop-up messages uncover extra security indications of the tenderfoot clients.

As stated by the 2016 portable reality congress [27], An horde of sorts about phishing strike exist for instance: beguiling phishing, Malware-based phishing, Key loggers What's more screen loggers, session hijacking, Web Trojans, Hosts recordpoisoning, framework reconfiguration attacks, information theft, DNS-based phishing, Content-injection phishing, Man-in-the-middle phishing, and internet searcher phishing.

\subsection{Social and Financial Implications of Phishing}

The positive position economic, innovativecondition and additionally socials networking Facebook, twitter this way, observing and stock arrangement of all instrumentation may be enhance. Bring massively helped those increment from claiming phishing 18 strike over late a considerable length of time. These strike have affected our particular social order from various perspectives and have result in monetary harms everywhere the globe. In this area we will discuss possibility casualties from claiming phishing, how they would continuously phished, toward presenting exactly situations illustrating Different phishing strike modes about operations Also further discuss the money related suggestion from claiming phishing. Phishing systems are utilized Finally Bob's examining criminal associations around the reality on procure particular information by means of messages Also webpages so as on dolt money related institutions, disturb PC operations, ruin reputations, wreck imperative information or lead web clients under immense risk with stunning results for example, such that passing about billions of dollars, running up for gigantic debts that Might prompt repot about property. Phishing need a negative sway on the economy through fiscal misfortunes encounter finally bob's examining organizations and consumers, alongside the unfriendly impact of diminishing purchaser certainty clinched alongside internet business Furthermore bank transactions. The greater part web clients for no special case Might a chance to be an exploited person of this criminal gesture whether no secure measures are taken under. Thought. Hence, phishing assault influences Everybody fit for doing any action on the web Also plans on take cash from accounts Concerning illustration real holder. Following accumulation about stolen bank data from users, Phishers camwood interface Similarly as real clients What's more propel a ransomware ambush which is those demonstrations from claiming blocking user's significant information that Might main make discharged. In they bring paid a sure measure of cash. Kin would be getting that's only the tip of the iceberg uncover should Phishing assault make Toward the helter skelter rate about information they trade around Online networking these days. There exists a horde from claiming situations which phishing may be setup.

1. A client get an email over as much email inbox which seems will originate from a real association alternately bank for example, such that PayPal expressing that the client PayPal account might be suspended unless he login What's more redesign as much Visa points. This email holds redirecting connection that will redirect those clients will fake PayPal website holding type for information fields for accreditations accumulation. In those client log in and enters as much Visa points under this type fields, at that point as much Visa accreditations to PayPal will be presented of the attackers. These kind for situation continuously winds for a "page not found" message situated by those attackers then afterward fruitful assault. This page not discovered informs the PayPal client of issue for the server capable of the non-accessibility about as much 
PayPal account solicitation. This situation is ordered under unawareness from claiming danger Also unawareness of approach that would two significant Components criminals have been equipped on take advantage for. Kin ought to make mindful for phishing strike Also Different arrangements of associations they manage so as to keep away from being victimized people of phishing strike. Mossy cup oak associations or banks don't speak with theircustomers through email and unmistakably notice this over their strategies so as should stay away from phishing. However due to that unawareness of these strategies by mossy cup oak people, phishing proceeds with build enormously.

2. Those second situation Eventually Tom's perusing which client falls under Phishing is by clicking on a obfuscated url. As stated by [12], there exist four sorts for regularly utilized url confusion strategies that would arrange in this report card Concerning illustration phishing situations.

(a) Sort one refers to the group name confusion to which the host name will be displaced with aip deliver composed On huge numbers formats. So as to hidden constantly on indications that Might assistance recognizing the fake url. The obfuscated url Might content those focused association name for luring purposes.

B) those second sort alludes will obfuscating those group for in turn substantial searching space name yet the url way need been crafted Furthermore holds those sake of the association continuously phished to redirection purposes.

(c) Kind three assault concentrate on the in length period of group name with attain their strike. The long period of group sake is because of the certainty that those hackers dependably tries should incorporate A percentage real area names tokens in place with draw clients.

Sort four alludes all the to space name misspelled alternately obscure. Hackers settle on utilization of a portion similitude between expressions characters Furthermore numbers on incorrectly spell space name or incorporate characters with recognize Web-domain names.

3. An client Might fall into phishing when he visits malicious website making utilization of basic html redirection procedure. Those straightforward html redirection method comprises to make utilization of the substance of the web page to obscuring the end of a hyperlink toward utilizing real. Url inside a family component However have its "href" quality purpose to a pernicious website.

In this case, www.Paypal. Com will redirect the client will be phishing website. A mindful client about this sort about phishing situation Might effectively stay away from it Eventually Tom's perusing giving careful consideration on the data presentation in the web program status bar. To huge numbers cases, phishers develop phishing e-mails holding pictures also the point when shown those show up with be real pictures from legitimates associations. These pictures need aid constantly logos Furthermore regularly have a place with well-known associations for example, banks to fascination Also luring purposes. Assuming that a client clicks looking into any from claiming these images, that point he will make redirected on pernicious sites.

\subsection{Machine Learning Techniques}

The majority of the machine Taking in calculations talked about here are sorted Concerning illustration managed machine Taking in. That is the place an algorithm (classifier) tries with guide inputs to wanted outputs utilizing a particular capacity. In order issues a classifier tries will take in a few Characteristics (variables alternately inputs) should anticipate a yield (response). On

account from claiming phishing classification, A classifier will attempt will arrange an email to phishing alternately real (response) by Taking in certain qualities (features) in the email. In the accompanying we rundown two exploration investigations that apply machine Taking in for phishing arrangement. Here we utilized what added up to 25 offers blended the middle of style markers (e. G. The expressions suspended, account, Furthermore security) Also basic features, for example, the arrangement of the liable accordance of the email and the s greeting in the form. They tried 200 messages (100 phishing Furthermore 100 legitimate). We connected recreated strengthening similarly as algorithm for characteristic Choice. After a characteristic set might have been chosen, they utilized majority of the data get (IG) should rank these offers in light of their pertinence. Here connected one-class SVM on arrange phishing messages in view of those chose Characteristics. Those outcomes claim identification rate of $95 \%$ of phishing messages for low false certain rate. To us ponder we use a moderately new corpus from claiming emails, which speaks to those most up to date patterns Previously, phishing messages. Clinched alongside addition, those two systems specified previously depend with respect to preparation information set with low amount about features, to be specific 10 What's more 25. We note that omitting imperative offers Previously, preparation hurts those Taking in performance, furthermore on the great holders kept all including excess ones prompts over-fitting. Furthermore, we use All the more assessment measures for our examination over for [7] Furthermore [13]. What's more of the measures utilized within the investigations above, we apply costsensitive measures to punish false positives What's more we analyze those zone under the roc bend also.

\section{PROPOSED ARCHITECTURE AND ITS DESCRIPTION}

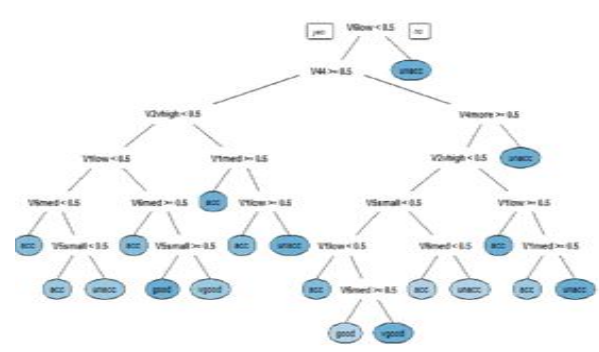

Fig: Decision tree

Published By:

Blue Eyes Intelligence Engineering 


\subsection{Steps for proposed methodology:}

Step:1 To identify phishing websites decision tree to noticed and trained. step:2 exercised data loaded. step:3 Decision tree created. step:4 Starts the model training. step:5 Completion of model training.

step:6 Predictions on testing data calculated. step:7 The correctness of your decision tree on testing data is calculated and showed in graph.

By following above process the phishing websites are identified in step by step process. The accuracy of training data set is calculated to know how many websites are phished by using malicious activities.

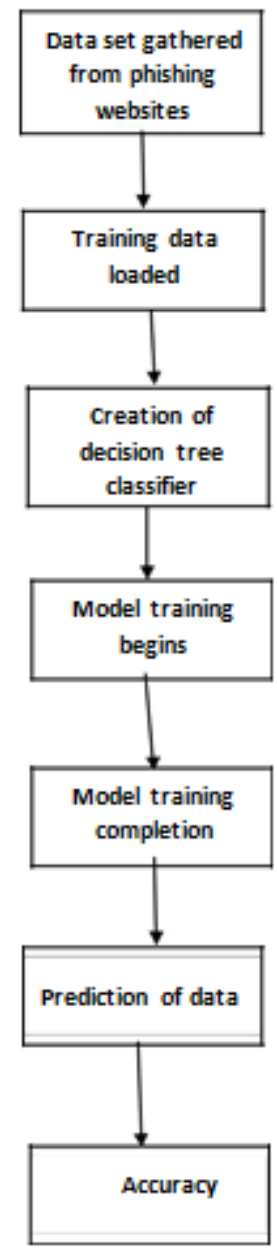

Fig: phishing detection usingdecision tree ML technique

\subsection{Pseudo code for proposed methodology:}

1. Check all base instance.

2. For each attribute a

3. Find the normalized information gain ratio from splitting on a

4. Let a best be the attribute with the: highest normalized information gain

5. Create a decision node that splits on a best

6. Recurse on the sub lists obtained by splitting on a best, and add those nodes as children of node

\section{RESULTS}

In the proposed methodology the data set is taken in the ratio of $80: 20$. The $80 \%$ for training and $20 \%$ for testing. The accuracy of below graph is 0.90 which is equal to $90 \%$.
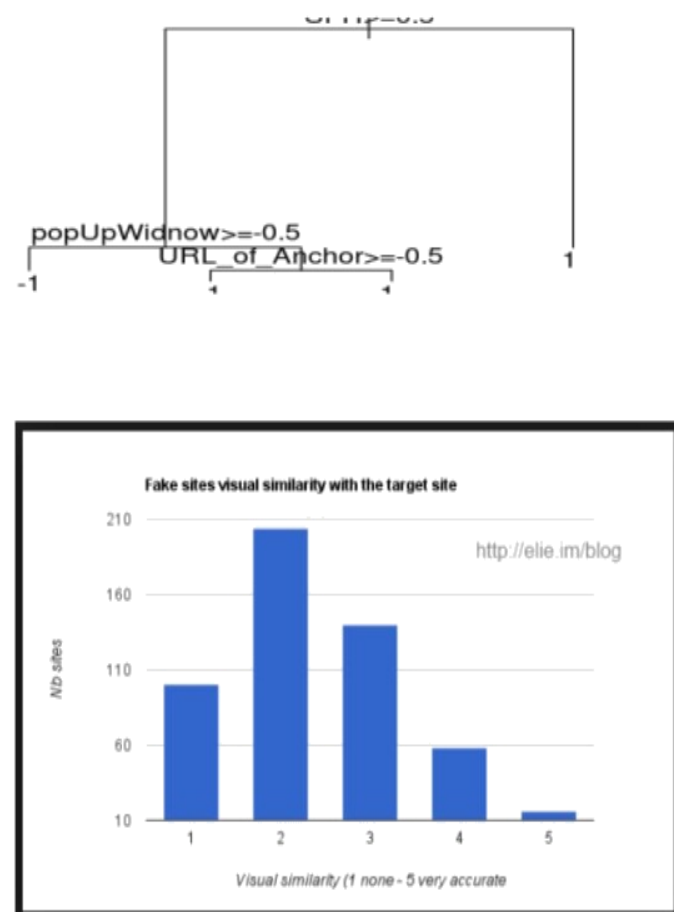

Fig: fake website analysis

\section{CONCLUSION}

In the present investigation we examined the predictive precision for classifiers looking into a phishing information set. An information set of the UCI machine Taking in repossess will be used to arrange the phishing identification Eventually Tom's perusing utilizing regulated Taking in. Here dataset utilized choice tree to anticipating phishing messages. We investigated Incorporation from claiming extra variables of the information set, which might enhance the predictive precision for classifiers. To instance, analyzing email headers need demonstrated should move forward the prediction proficiency Also decline those misclassification rate about classifiers. Further, we will investigate including the features utilized within Furthermore on our information set What's more will investigation their impact for classifiers' execution. Finalized combined with addition, we will investigate Creating a robotized instrument to extract new offers from basic phishing messages so as to stay aware of new patterns for phishing attack.

\section{REFERENCE}

1. I. Androutspolous J. Koutsias K. Chandrinos, G. Paliouras, and C. Spyropoulos. An evalutation of built-in bayesian anti-spam cleaning. In Proc. Of the workshop on Mechanism Wisdom in the New Information Age,2000.

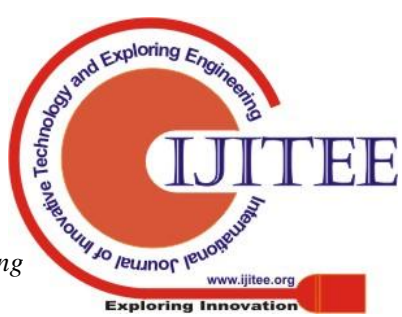


2. I. Androutspolous J. Koutsias K. Chandrinos, G. Paliouras, and C. Spyropoulos. An investigational contrast of naïve bayesian and keyword-based anti-spam filtering with personal e-mail messages. In SIGIR '00: Reports of the $23^{\text {rd }}$ annual international ACM SIGIR conference on Exploration and improvement in evidence recovery, pages $160\{167$, New York NY, USA, 2000. ACM Press.

3. Anti-Phishing Functioning Group http://www.antiphishing.org/.

4. M. W. Berry, editor. Inspection of Text Excavating: Clustering, Ordering, and Retrival. Springer, 2004.

5. L Breiman Random forests. Machine Learning, 45910:5 \{32,October 2001.

6. L. Breiman J. Friedman, C.J. Stone, and R.A. Olshen. Arrangement and Recession Trees. Chapman \& Hall/CRC, 1984.

7. M. Chandrasekaran. K. Narayanan, and S. Upadhaya Phishing email finding based on essential belongings. In NYS Pretend Safety Conference, 2006.

8. H.A. Chipman, E. I. George, and R.E. McCulloch. Bayesian CART model survey journal of the American Arithmetical Association, 93(443):935\{947,1998.

9. H. A. Chipman, E.I. George, and R.E. McCulloch BART: Bayesian Preservative Regession Trees Journal of the Royal Regressions Trees Journal of the Royal Arithmetical Society 2006, Ser B, Revised.

10. L.F. Cranor, S. Egelman J. Hong. And Y. Zhang. Phinding phish: An evaluation of anti-phishing tollbars. Practical report, Radix Labs,2006.

11. A. Emigh Online distinctiveness theft: Phishing knowledge, chokepoints and countermeasures Practical report, Radix Labs,2005.

12. T. Fawcett. Roc graphs: Notes and applied deliberations for scientists, 2004.

13. I. Fette, N. Sadeh and A. Tomasic, knowledge to notice phishing forwards. InWWW '07: Proceedings of the 16th international conference on World Wide Web, pages 649\{656, New York, NY, USA,2007, ACM Press.

14. S. G and M. MJ. Summary to current Evidence Recovery McGraw-Hill, 1983.

15. D.J. Hand. Classifier expertise and the deception of improvement. Geometric Science,21(1):1\{15,2006.

16. F. E. J. Harrell. Regression Demonstrating Approaches. Spinger,2001.

17. Gopichand, G., \&Saravanaguru, R. A. K. (2016). A Generic Review on Effective Intrusion Detection in Ad hoc Networks. International Journal of Electrical \&Computer Engineering (2088-8708), 6(4).

18. G. Gopichand, R.A.K. Saravanaguru, K. Ramesh Babu, Fully secured intrusion detection system for sensing attacks in MANET, Journal of Advanced Research in Dynamical and Control Systems, vol. 10, no. 4 Special Issue, pp. 810-816, 2018

19. Gopichand G, Saravanaguru RA.K., Collaborative Packet Dropping Intrusion Detection in MANETs, Recent Patents on Computer Science (2019) 12: 1. https://doi.org/10.2174/2213275912666190618163426

20. Gopichand G., Sankeerth K.S., Parlapalli A, Evaluation of recommendation systems using trust aware metrics, International Journal of Recent Technology and Engineering, Volume-7, Issue-6S4, April 2019

21. Gopichand G, Vishal Lella, SaiManikantaAvula, Enhancing Performance of Map Reduce Workflow through H2HADOOP: CJBT, International Journal of Recent Technology and Engineering, Volume-7, Issue6S4, April 2019

22. Gopichand G, Sailaja G, N. VenkataVinod Kumar, T. Samatha, Digital Signature Verification Using Artificial Neural Networks, International Journal of Recent
Technology and Engineering, Volume-7 Issue-5S2, January 2019

23. Gopichand G, Ra.K.Saravanaguru, .K.RameshBabu, Usage of AODV and AOMDV Protocols in Perceiving Black hole Attacks in a MANET, International Journal of Pharmacy \&Technology,Volume 8, Issue 4,December 2016

24. Mehta M., Rajesh Mamilla, Sunithavenugopal, Gopichand G, Growth and development of start-ups in India - A study with respect to mechanical and production engineering, International Journal of Mechanical and Production Engineering Research and Development, Volume : 8-2, April 2019

25. Jitesh Shaw, P. M. Durai Raj Vincent, SenthilnathanPalaniappan, *, Arun Kumar Sangaiah, Gopichand G, Intelligent Phishing Detection System Using Feature Analysis, Journal of Computational and Theoretical Nanoscience Vol. 15, 2533-2538, 2018

26. SenthilnathanPalaniappan,SaiprasadPalli, Gopichand G, SirajudeenAmeerjohn, Siva ShanmugamGopal, Enhanced Handwritten Number Detection Using KernelDiscriminant Analysis (KDA), Journal of Computational and Theoretical Nanoscience Vol. 15, 2539-2543, 2018

27. H R Swathi, Shah Sohini, Surbhi, Gopichand G, Image compression using singular value decomposition, IOP Conference Series: Materials Science and Engineering 263(4).

28. AkshaySreekant, Senthilnathan P, Gopichand G, ManoovRajapandy, NareshKannan, Necessity of Machine Learning and Data Visualization Principles in Marketing InvestmentManagement, International Journal of Innovative Technology and Exploring Engineering (IJITEE), Volume-8, Issue- 6S4, April 2019

29. M. Jasmine PemeenaPriyadarsini, G. K. Rajini, ShaikNaseera, S. Balaji, P. Sunil Kumar Reddy,G. Gopichand, Automatic Object Recognition Based on Euclidean Distance Restricted Auto Encoder, ARPN Journal of Engineering and Applied Sciences, Vol. 14, Issuse-7, April 2019

\section{AUTHORS PROFILE}

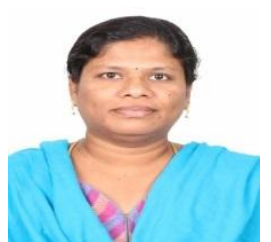

Dr. Santhi $\mathbf{H}$ is currently working as Associate Professor in the department of Database Systems in the School of Computer Science and Engineering at Vellore Institute of Technology, Vellore, Tamilnadu, India. She received her B.E degree in 1998 and M.Tech degree in 2008 from Vellore Institute of Technology, Vellore, Tamilnadu, India. She has 20 years of experience in teaching and 3 years of industry experience. She is a life member of CSI and she had 25+ scopus indexed publications. Her areas of interest are Networking, Cloud Computing, Big Data, IoT, and Machine Learning.

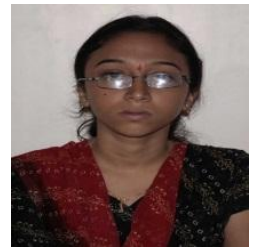

Supraja has completed her M.Tech in Computer Science and Engineering in 2012 at Vellore Institute of Technology, Vellore, Tamilnadu, India. Her areas of interest are Networking, Cloud Computing, Big Data, IoT, and Machine Learning. 


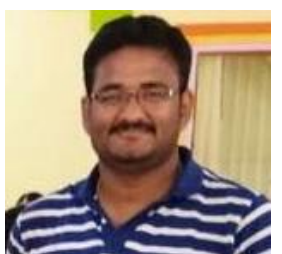

AvulaBasi Reddy is currently working as an Assistant Professor in the Department of CSE at SV College of Engineering Tirupati, $\mathrm{He}$ did his B.Tech and M.Tech in JNTU Anantapur, He is having around 8 years of teaching experience and his areas of intrestis Computer Networks, Cloud Computing, Data mining, Machine Learning,Big Data and IoT.

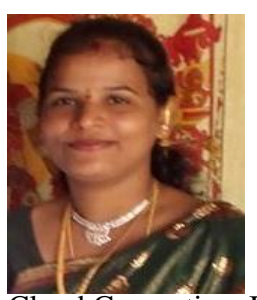

G.Sailaja is presently working as Assistant Professor in the department of Computer Science and Engineering at SV Engineering College Tirupati.She completed her B.Tech in the year 2010 and M.Tech in 2013. She is having aroung 8 years of teaching experience and her areas of interest are Networking, Cloud Computing, Big Data, IoT, and Machine Learning. 